\title{
Facial Paralysis in Children
}

\author{
Sashank Reddy, MD, $\mathrm{PhD}^{1} \quad$ Richard Redett, $\mathrm{MD}^{1}$ \\ ${ }^{1}$ Department of Plastic and Reconstructive Surgery, Johns Hopkins \\ School of Medicine, Baltimore, Maryland \\ Facial Plast Surg 2015;31:117-122.
}

\begin{abstract}
Address for correspondence Richard Redett, MD, Department of Plastic and Reconstructive Surgery, Bloomberg 7314b, Johns Hopkins School of Medicine, 1800 Orleans St., Baltimore, MD 21287 (e-mail: rredett@jhmi.edu).
\end{abstract}
Abstract
Keywords
- facial paralysis
- pediatrics
- facial nerve

\begin{abstract}
Facial paralysis can have devastating physical and psychosocial consequences. These are particularly severe in children in whom loss of emotional expressiveness can impair social development and integration. The etiologies of facial paralysis, prospects for spontaneous recovery, and functions requiring restoration differ in children as compared with adults. Here we review contemporary management of facial paralysis with a focus on special considerations for pediatric patients.
\end{abstract}

\section{Basic Science}

Appropriate diagnosis and management of facial paralysis begins with a detailed understanding of facial nerve anatomy. Upon exiting the brainstem, the facial nerve travels intratemporally through the internal auditory meatus. The serpentine course of the nerve here renders it vulnerable to shear stress that can result in neuropraxia, axonotmesis, or avulsion. The nerve is also subject to laceration by temporal bone fragments in skull base fractures. The three intratemporal branches of the facial nerve include the greater petrosal nerve, which conveys parasympathetic information, the motor nerve to the stapedius, and the chorda tympani that relays taste sensation from the anterior two-thirds of the tongue in addition to its parasympathetic functions. Extratemporal preparotid branches include the posterior auricular branch as well as branches to the digastric and stylohyoid. The facial nerve exits the stylomastoid foramen in a more superficial position in utero and in neonates than it does in adults. This makes it susceptible to compression by the maternal pelvis or during delivery. ${ }^{1}$ Because of its superficial location, the extratemporal facial nerve is also more prone to iatrogenic injury during facial surgery on infants or young children. Within the parotid the main branches of the nerve are frontal, zygomatic, buccal, marginal mandibular, and cervical. In practice there are frequent interconnections and redundancies in these branches particularly zygomatic and buccal. Knowledge of facial nerve topography is useful in localizing lesions and planning repair.

Several studies have reported improved regeneration of peripheral nerves in children compared with adults. Both median and ulnar nerve repairs, for example, have had superior outcomes in children. ${ }^{2,3}$ Similar results have been noted in facial nerve regeneration. One study of chorda tympani repair found markedly better regeneration and recovery of taste function in pediatric patients $(66.7 \%)$ versus adults (31.6\%). ${ }^{4}$ This corresponded to objectively improved electrogustatory thresholds. Improved regeneration in children coupled with their greater cortical plasticity leads to improved outcomes in most cases of facial paralysis. ${ }^{5}$

\section{Etiology}

Facial paralysis in children results from acquired or congenital causes. Acquired causes include infection, Bell palsy, trauma, and neoplasm. In one multiyear review of facial paralysis at Boston Children's Hospital, the single largest cause was infection, with the majority of these being complications of acute otitis media. ${ }^{6}$ Other infectious causes of facial paralysis include herpes simplex virus, Epstein-Barr virus, human immunodeficiency virus, cytomegalovirus, mumps, and rubella.

In the neonatal period, traumatic facial nerve paralysis is most commonly a sequela of delivery. Difficult extractionparticularly accompanied by the use of birth forcepsincreases the risk of facial nerve paralysis. ${ }^{7}$ The prospects for spontaneous recovery are excellent, however, with nearly $90 \%$ of patients exhibiting complete recovery and the remaining patients showing partial recovery in one survey. ${ }^{7}$ In older children, traumatic facial nerve injury can occur secondary to blunt trauma from sports or from motor vehicle accidents. ${ }^{6}$ Again, spontaneous recovery can be expected with a mean
Issue Theme Current Concepts, State of the Art Treatment of Facial Paralysis; Guest Editor, Patrick J. Byrne, MD, FACS
Copyright (c) 2015 by Thieme Medical Publishers, Inc., 333 Seventh Avenue, New York, NY 10001, USA. Tel: +1(212) 584-4662.
DOI http://dx.doi.org/ 10.1055/s-0035-1549042. ISSN $0736-6825$. 
duration of several months. Penetrating injury to the facial nerve, while rarer in children, has a poor prognosis unless surgical repair is accomplished.

Developmental facial paralysis can occur as an isolated phenomenon or in association with other anomalies. Mobius syndrome is the most common developmental cause of bilateral facial palsy. It is defined by bilateral dysfunction of the facial and abducens nerves, leading to a characteristic mask-like faces with partial or complete facial palsy and inability to abduct the eyes. The etiology of Mobius syndrome is unclear, though aberrant vascular development has been cited as a possible inciting event. ${ }^{8,9}$ Hemifacial microsomia, an umbrella term for developmental anomalies involving the first and second branchial arches, is also associated with facial palsy. Studies have reported facial paralysis in 22 to $50 \%$ of people with hemifacial microsomia. Goldenhar syndrome is subsumed under the broader category of hemifacial microsomia and can also be associated with facial paralysis. Children with developmental facial paralysis frequently have other anomalies particularly those involving the limbs. ${ }^{10}$

\section{Approaches to Restoration}

Treatment strategies for facial paralysis are guided by those facial nerve functions needing restoration. Some deficits of facial nerve function including hyperacusis, decreased salivation, or impaired taste are not overly troublesome for patients or are adequately compensated by the contralateral, functioning nerve, and repair is usually not undertaken. Other, more critical functions to restore are divided by anatomic region. In the upper face eye closure is the priority, in the midface correction of facial ptosis and any nasal stenosis is important, and in the lower face symmetric smile and oral continence are critical.

The myriad of surgical options for children with facial paralysis can be divided into static procedures aimed at ocular protection and restoration of resting symmetry, and dynamic procedures aimed at true facial reanimation. In children every attempt must be made to restore facial movement and allow for social integration. Dynamic procedures include primary nerve repair, nerve conduit placement, repair with nerve grafting, cross-facial nerve graft with or without a babysitter procedure, neurotization procedures using other cranial nerves, regional muscle transfer, and free muscle transfer. Choosing the optimal strategy depends principally on three factors: (1) etiology of the facial paralysis (congenital versus acquired), (2) location of the facial nerve deficit (proximal vs. distal, intratemporal vs. extratemporal), and (3) chronicity of paralysis. This last factor predominates in our planning, because the end muscles innervated by the facial nerve undergo irreversible atrophy and fibrosis after 12 to 18 months. Long-term facial paralysis therefore necessitates introduction of new muscle to restore animation. Furthermore, the slow speed of nerve regeneration, approximately $1 \mathrm{~mm} /$ day, may prevent complete reinnervation of motor endplates prior to end-organ degeneration, particularly in proximal injuries. Our focus on the status of the end organ in reconstructive planning has led us and others to term this the target-organ approach. ${ }^{11}$

\section{Management of Recent Deficits ( $<6$ months) -Primary Repair, Neurotization, Cross-Facial Nerve Grafting}

For treatment of early deficits of less than 12-month duration, every attempt is made to restore innervation to the native musculature. Methods used to accomplish reconstruction of recent facial nerve deficits are chosen based on the etiology of facial paralysis and on the location of the injury to the facial nerve. In neonates with facial paralysis from birth trauma, expectant management is preferred, as greater than $90 \%$ of patients can expect full recovery on the order of weeks to months. ${ }^{7,12-14}$ Interventions can be considered if patients do not have adequate return of function by 1 to 2 years of age although facial nerve surgery in this age group is not that common, and reconstruction is typically delayed until the child reaches 4 to 5 years ${ }^{15}$ (see - Fig. 4). For children with acute onset facial paralysis of idiopathic or infectious etiology, observation or medical management is the initial course of action. Idiopathic facial paralysis usually resolves in 6 to 12 months in the majority of patients. ${ }^{6}$ However, these patients need to be followed very closely to make sure recovery is complete and surgical intervention is not warranted. Children with facial paralysis from otitis media are treated with myringotomy, antibiotics, and possibly a course of steroids. ${ }^{6,16}$ Prospects for spontaneous recovery are good with most patients recovering following administration of antibiotics with a mean duration of paralysis of less than 1 month. $^{6}$

For recent facial paralysis due to anatomic disruption of the facial nerve, management is guided by the location of the injury-intratemporal, extratemporal and proximal, or extratemporal and distal. Extratemporal injuries to major branches should be repaired in a tension-free manner either primarily, with a nerve conduit, or with the aid of a nerve graft. Primary nerve repair is accomplished by joining the proximal and distal ends of the injured nerve using microsurgical techniques with fine nylon suture or tissue glue. When a nerve gap precludes primary repair or imposes excessive tension, nerve conduits or nerve grafts are used. Gaps of less than $3 \mathrm{~cm}$ are amenable to either approach. In animal models, the use of a bioresorbable nerve conduit was shown to be either equivalent or superior to nerve grafting for peripheral nerve repairs. ${ }^{17,18}$ For the facial nerve specifically, repair of distal lesions with nerve conduits has shown good to excellent muscle recovery in $70 \%$ of cases. ${ }^{19}$ Nerve grafting can also lead to excellent results (-Figs. 1 and 2 ), and the choice is ultimately guided by surgeon preference and availability of donor tissues.

The poorly defined fascicular topography of the facial nerve proximal to the stylomastoid foramen has discouraged surgeons from attempting direct repairs after injury. Several studies have demonstrated dyskinesis or synkinesis with such repairs, leading many to advocate cross-facial nerve grafting or alternative techniques. ${ }^{20,21}$ These studies 


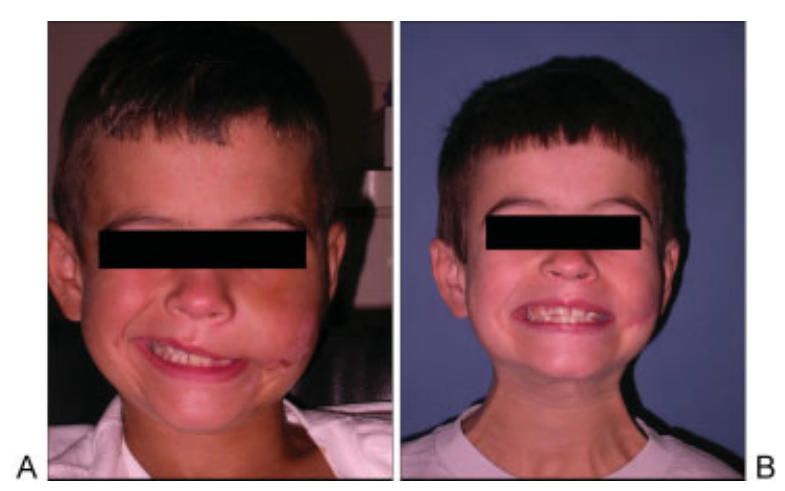

Fig. 1 Acute repair of facial nerve trauma with sural nerve grafts. (A) Sural nerve grafts were performed to upper, middle, and lower facial nerve branches on a 6-year-old child 1 month after he had traumatic disruption of the facial nerve in an all-terrain-vehicle accident. (B) Eighteen months later he had nearly perfect symmetry of his smile.
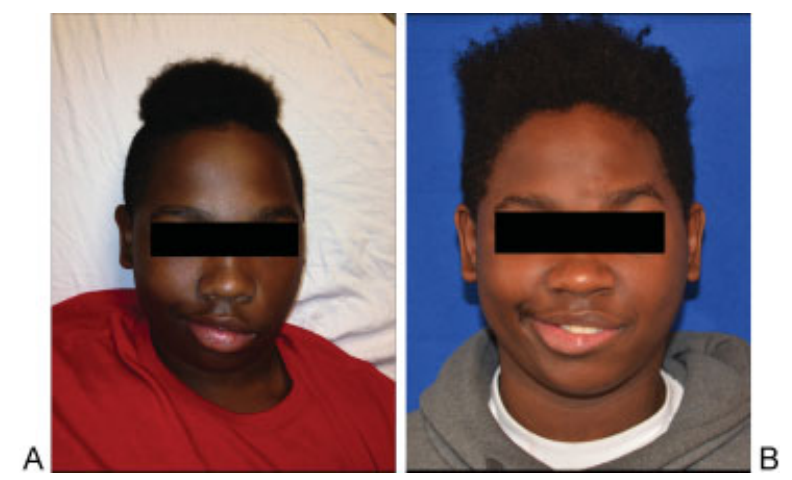

Fig. 2 (A) Nerve graft repair of ballistic injury to facial nerve trunk. Nerve grafting was performed on a 10 -year-old patient who suffered a gunshot wound to the facial nerve trunk proximal to the stylomastoid foramen. Following partial mastoidectomy, facial nerve exploration revealed destruction of the proximal trunk. Distal segments were coapted to the trunk using sural nerve grafts. (B) At 11-month followup the child has excellent symmetry with spontaneous smile and minimal synkinesis.

generally considered adult patients, however, so their generalizability to young children is uncertain. Anecdotally, we have achieved good restoration of facial muscle function with minimal synkinesis in children with this type of injury. It is likely that the greater cortical plasticity of children in part accounts for the improved results with this approach, but more examples are needed before it is considered a standard first option for proximal lesions. ${ }^{22,23}$

Cross-facial nerve grafting is the mainstay of management for early intratemporal injuries. Using a modified facelift type of incision, sural or peroneal nerve grafts are coapted to the corresponding branches on the uninjured side and guided across the face in the subcutaneous plane where the ends of the graft are marked with fine Prolene sutures. We target the facial nerve branch to the zygomaticus major (smile), orbicularis oculi (eye closure), and frontal branch (eyebrow elevation) when possible. We generally do not target the marginal mandibular nerve as selective myotomy of the depressor anguli oris at a later date is an effective procedure for symmetry. The motor branch to the zygomaticus major can be found at a Zuker point, which is half-way between the root of the helix and the commissure. ${ }^{24}$ Given the time it takes for regenerating axons to grow through the cross-facial nerve graft and reach the contralateral side (up to 6 months), there is a risk that the target muscles will have undergone denervation atrophy. To avoid this, many surgeons perform a "babysitter" procedure in which a partial hypoglossal or masseteric motor nerve transfer is coapted to the injured ipsilateral facial nerve. The hypoglossal or masseteric motor nerve grows into the facial musculature, thereby preventing muscle atrophy and fibrosis. When distal regeneration of the cross-facial nerve grafts is confirmed by Tinel sign, the babysitter nerve connections are taken down and coaptations of the cross-facial nerve graft are performed to facial nerve branches supplying zygomaticus major, orbicularis oculi, and the frontal branch. In a review of 20 patients who underwent babysitter procedures over a nearly 20 -year period, Terzis and Tzafetta reported that $75 \%$ of patients achieved good to excellent functional and aesthetic outcomes. ${ }^{25}$ As would be predicted, the duration of facial paralysis correlated with ultimate outcome, with patients having less than 12 months of paralysis achieving superior results. Additional cosmetic procedures for rebalancing, if necessary, are performed after full adaptation of the patient to the graft has taken place in 12 to 24 months.

\section{Management of Delayed Deficits (6-12 months)-Primary Nerve Repair, Neurotization, Cross-Facial Nerve Grafting}

Patients who present in a delayed fashion between 6 and 12 months after facial nerve injury can benefit from primary nerve repair if the injury is extratemporal, cross-facial nerve grafting as described previously, or neurotization. By 6 to 12 months after injury, muscle atrophy and fibrosis is underway and quick axonal input is needed for recovery. All facial nerve injuries that are extratemporal can be repaired primarily or with nerve grafts or conduits. For intratemporal or brainstem-level injuries, neurotization using other cranial nerves can be performed. Neurotization entails coapting a branch of another cranial nerve-typically the hypoglossal or the trigeminal motor branch to the masseter-to distal branches of the facial nerve. The motor branch to the masseter is particularly useful in this regard as it contains on average more than 1,500 axons, which compares favorably to 100 to 200 axons present at the end of a cross-facial nerve graft. ${ }^{26}$ The buccal branch of the facial nerve has approximately 800 axons. Through a small facelift incision, the recipient branches of the facial nerve are identified. The motor nerve to the masseter muscle is located $1.5 \mathrm{~cm}$ inferior to the zygomatic arch and $1.5 \mathrm{~cm}$ anterior to the posterior border of the muscle in adults, and can be found in a corresponding position in children. ${ }^{11}$ The nerve is then dissected free from the deep layers of the muscle, divided, and then coapted to the facial nerve trunk or distal branches. ${ }^{26}$ When using this technique, we target the motor branch to the zygomaticus major and levator. This procedure can produce strong, 
volitional lip and commissure elevation after several months but requires the patient to gently bite down to achieve movement. In small children there is a possibility for spontaneous, effective muscle movement following masseter nerve transfer. ${ }^{5,27}$ Intensive physical therapy with biofeedback can be important in gaining spontaneous movement. ${ }^{28}$ The hypoglossal nerve is a proven alternative to the masseteric nerve for neurotization, but its harvest can be complicated by hemilingual atrophy and tongue disfunction. ${ }^{29}$

\section{Management of Long-Term Deficits (>12 months)-Free Tissue Transfer}

In patients with facial paralysis of greater than 12-month duration, the target musculature will have undergone irreversible atrophy and fibrosis. This necessitates the introduction and innervation of new muscle to restore dynamic movement. The first report of free tissue transfer for facial reanimation is credited to Harii et al in $1976 .{ }^{30}$ He performed gracilis transfer in a patient with long-standing Bell palsy, using the ipsilateral nerve to the temporalis for coaptation. The patient had a marked improvement and could smile volitionally while biting down, but spontaneous smile was not attained. Building on this pioneering work, contemporary microsurgical approaches to facial reanimation enable spontaneous, emotive expression. Our preferred approach requires three stages: (1) cross-facial nerve graft, (2) free tissue transfer, (3) cosmetic balancing procedures. We initially perform a short cross-facial nerve graft that is banked above the canine tooth through a small incision made in the gingivobuccal sulcus and marked with 5-0 Prolene suture. At the time of gracilis transfer, the motor branch is positioned in the upper lip near the previously marked graft and the nerve coaptation is performed after reopening the gingivobuccal sulcus incision. The use of this shorter cross-facial nerve graft reduces the distance regenerating axons have to travel, thereby increasing the axon density at the distal end. An alternative to cross-facial nerve grafting is powering the transferred muscle using the motor nerve to the masseter muscle, but, as with Harii's original procedure, this does not always achieve spontaneous, emotive expression. ${ }^{26} \mathrm{We}$ reserve this for cases of bilateral facial nerve paralysis such as that occurring in Mobius syndrome or when a prior crossfacial nerve graft has failed. Harii has recently described taking a longer segment of the motor nerve to the latissimus and coapting it to the uninjured, contralateral facial nerve in a single stage. In the reported 15-year experience, functional outcomes are comparable to two-stage gracilis transfer. ${ }^{31}$

Free muscle transfer is accomplished approximately 9 to 12 months after the first stage when the Tinel sign confirms that axons have reached the end of the graft. The gracilis is our first choice flap for facial reanimation, but the latissimus dorsi and pectoralis minor have been utilized as well. ${ }^{32-35}$ We use a two-team approach to allow muscle harvest and preparation of the recipient site in order to proceed simultaneously. To minimize bulk in the face, approximately $50 \%$ of the gracilis is harvested with the aid of a gastrointestinal anastomotic stapler or a line of sutures to reinforce the end of the muscle. ${ }^{11}$ The length of muscle harvest is determined by the distance from the preauricular region to the commissure plus $1 \mathrm{~cm}$. The muscle is transferred to the face and carefully inset to ensure appropriate tension and direction of pull (-Figs. 3 and 4). Vascular anastomosis is performed to facial vessels when available. Nerve coaptation then takes place with alignment of fascicles from the obturator anterior branch to the gracilis and fascicles of the previously banked nerve graft. Revisional surgeries, when necessary, are performed one to 2 years after free muscle transfer. These include muscle tightening or repositioning, debulking, face lift, blepharoplasty, brow lift, and Botox injection. Early outcomes tend to be good as free flaps are reinnervated more quickly in children. ${ }^{36}$ Terzis and Olivares have examined long-term outcomes in children who have undergone free muscle transfer for facial reanimation. Their study of patients followed from between 5 and 22 years found that they maintained their degree of reanimation and resting symmetry over time and that the gracilis continued to grow with the craniofacial skeleton. ${ }^{37}$

In patients who cannot undergo free tissue transfer, regional muscle transfers remain an option to restore dynamic facial movement. The temporalis transfer, in which the muscle is disinserted from the mandible and redirected to the oral commissure, is a long-standing procedure for facial reanimation. It has numerous disadvantages, though, which limit its use to situations in which free tissue transfer is precluded or has already failed. These include lack of spontaneous, effective movement, temporal hollowing, and an
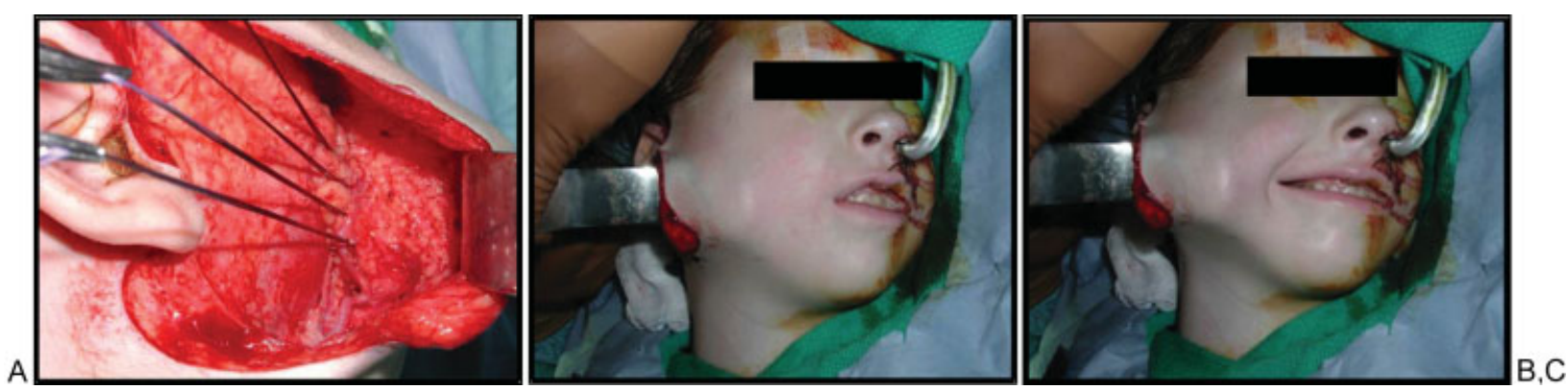

Fig. 3 (A) Inset of free gracilis for facial reanimation. A single Tycron suture is placed on the commissure, followed by two sutures at the upper lip and one at the lower lip. (B and C) The muscle position is then adjusted to establish a natural nasolabial crease at rest and symmetric smile with motion. Once the appropriate vector and tensioning are established, the muscle is inset superiorly on the SMAS with Tycron suture. 


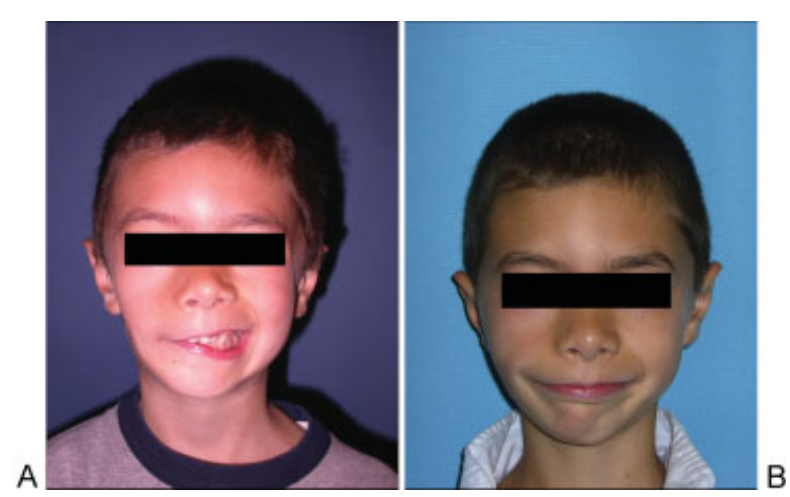

Fig. 4 Cross-facial nerve graft and gracilis transfer on a child with congenital facial palsy. (A) The patient failed to improve with expectant management and was treated with a cross-facial nerve graft at 5 years of age. This was followed by partial gracilis transfer 12 months later (B) At 2-year follow-up the child has a symmetric, spontaneous smile.

unnatural vector of smile. Boahene and colleagues have reported a minimally invasive temporalis transfer procedure that has less donor site morbidity than the conventional technique and can achieve some degree of spontaneous smile with intensive physiotherapy. ${ }^{38}$

\section{Ancillary Procedures}

Additional procedures can be used to address lip depression and provide blink restoration in patients with global facial paralysis. Failure of lip depression in particular can lend an unnatural appearance to the smile even after elevation has been restored. The simplest solution is to perform a balancing procedure to paralyze the contralateral lip depressors. This can aid in achieving lip symmetry both at rest and during smile. ${ }^{39}$ The depressor labii inferioris is located by asking the patient to show his or her lower teeth while the surgeon resists lip depression with an examining hand. The point of maximal resistance marks the depressor. Twelve units of Botox are introduced into the depressor along its mid-lateral border. If results are good, chemodenervation can be repeated as needed or the muscle can be resected through a small intraoral incision for a permanent solution. A potentially superior approach is to restore lip depression to the paralyzed side. Terzis and colleagues have described dynamic restoration of depression by performing platysma or digastric muscle transfer, or by utilizing nerve transfer of the hypoglossal nerve to the cervicofacial branch of the ipsilateral facial nerve. $^{40}$

The other challenge for children with complete facial paralysis is restoring spontaneous blink. At a minimum, ocular protection from evaporation and external insults must be provided. Fortunately, the greater tautness of eyelid skin in children allows for adequate protection of the cornea with lubrication alone and does not usually necessitate gold weights or other static procedures unless complete paresis of the orbicularis oculi is present. ${ }^{41}$ A spontaneous blink is required, however, to allow for a normal facial appearance.
This is best achieved by restoring innervation to the paralyzed orbicularis through neurorrhaphy or nerve grafting of the temporal branch or by cross-facial nerve grafting when the proximal stump is unsuitable. ${ }^{42}$ These options are not viable for long-standing paralysis, however, which requires introduction of new motor units. Frey and colleagues have used a split gracilis with fascicles supplying blink function separately innervated by a dedicated cross-facial nerve graft from the contralateral zygomatic branch. ${ }^{4,44}$ This was the authors' preferred technique for blink restoration in children. It allowed all patients to achieve good orbicularis excursion and freed them from ointments or eye chambers for ocular protection. Similarly, Terzis and colleagues were able to restore coordinated spontaneous blink in children using cross-facial nerve grafting to pedicled frontalis or to free platysma slips. ${ }^{42}$ In combination, these advanced microsurgical approaches to restoring reflexive blink and spontaneous, affective smile bring us ever closer to the elusive normal in patients with global facial paralysis.

\section{Conclusion}

Modern management of the child with facial paralysis begins by considering etiology, location, and duration of paralysis and designing a customized solution. Attention to the duration of deficit in particular aids in choosing among the numerous options for facial reanimation. Whichever option is chosen, the superior nerve regeneration and cortical plasticity in small children provides optimism for good long-term outcomes. The ongoing development of techniques for dynamic blink and lip depression should enable more global solutions for children with facial paralysis and allow them to achieve flourishing lives.

\section{References}

1 Terzis JK, Anesti K. Developmental facial paralysis: a review. J Plast Reconstr Aesthet Surg 2011;64(10):1318-1333

2 Tajima T, Imai H. Results of median nerve repair in children. Microsurgery 1989;10(2):145-146

3 Baysefer A, Izci Y, Akay KM, Kayali H, Timurkaynak E. Surgical outcomes of ulnar nerve lesions in children. A retrospective clinical study. Pediatr Neurosurg 2004;40(3):107-111

4 Saito T, Yamada T, Okamoto M, Narita N. Comparison of regeneration of the chorda tympani nerve and gustatory function recovery after severing the nerve between pediatric and adult patients. Otology \& neurotology: official publication of the American Otological Society, American Neurotology Society. Otology \& Neurotology 2012;33(9):1567-1572

5 Lifchez SD, Matloub HS, Gosain AK. Cortical adaptation to restoration of smiling after free muscle transfer innervated by the nerve to the masseter. Plast Reconstr Surg 2005;115(6):1472-1479, discussion 1480-1482

6 Evans AK, Licameli G, Brietzke S, Whittemore K, Kenna M. Pediatric facial nerve paralysis: patients, management and outcomes. Int J Pediatr Otorhinolaryngol 2005;69(11):1521-1528

7 Falco NA, Eriksson E. Facial nerve palsy in the newborn: incidence and outcome. Plast Reconstr Surg 1990;85(1):1-4

8 Leong S, Ashwell KW. Is there a zone of vascular vulnerability in the fetal brain stem? Neurotoxicol Teratol 1997;19(4): 265-275 
9 Bavinck JN, Weaver DD. Subclavian artery supply disruption sequence: hypothesis of a vascular etiology for Poland, KlippelFeil, and Möbius anomalies. Am J Med Genet 1986;23(4):903-918

10 Bergstrom L, Baker BB. Syndromes associated with congenital facial paralysis. Otolaryngology-head and neck surgery: official journal of American Academy of Otolaryngology. Head Neck Surg 1981;89(2):336-342

11 Rosson GD, Redett RJ. Facial palsy: anatomy, etiology, grading, and surgical treatment. J Reconstr Microsurg 2008;24(6):379-389

12 Toelle SP, Boltshauser E. Long-term outcome in children with congenital unilateral facial nerve palsy. Neuropediatrics 2001; 32(3):130-135

13 Al Tawil K, Saleem N, Kadri H, Rifae MT, Tawakol H. Traumatic facial nerve palsy in newborns: is it always iatrogenic? Am J Perinatol 2010;27(9):711-713

14 Duval M, Daniel SJ. Facial nerve palsy in neonates secondary to forceps use. Arch Otolaryngol Head Neck Surg 2009;135(7): 634-636

15 Hughes CA, Harley EH, Milmoe G, Bala R, Martorella A. Birth trauma in the head and neck. Arch Otolaryngol Head Neck Surg 1999;125(2):193-199

16 Elliott CA, Zalzal GH, Gottlieb WR. Acute otitis media and facial paralysis in children. Ann Otol Rhinol Laryngol 1996;105(1): 58-62

17 Weber RA, Breidenbach WC, Brown RE, Jabaley ME, Mass DP. A randomized prospective study of polyglycolic acid conduits for digital nerve reconstruction in humans. Plast Reconstr Surg 2000; 106(5):1036-1045, discussion 1046-1048

18 Diaz LM, Steele MH, Guerra AB, et al. The role of topically administered FK506 (tacrolimus) at the time of facial nerve repair using entubulation neurorrhaphy in a rabbit model. Ann Plast Surg 2004;52(4):407-413

19 Navissano M, Malan F, Carnino R, Battiston B. Neurotube for facial nerve repair. Microsurgery 2005;25(4):268-271

20 Myckatyn TM, Mackinnon SE. The surgical management of facial nerve injury. Clin Plast Surg 2003;30(2):307-318

21 Samii M, Matthies C. Indication, technique and results of facial nerve reconstruction. Acta Neurochir (Wien) 1994;130(1-4): 125-139

22 Clark JH, Burger PC, Boahene DK, Niparko JK. Traumatic facial nerve neuroma with facial palsy presenting in infancy. Otology \& neurotology: official publication of the American Otological Society, American Neurotology Society. Otol Neurotol 2010;31(5): 813-816

23 McMonagle B, Al-Sanosi A, Croxson G, Fagan P. Facial schwannoma: results of a large case series and review. J Laryngol Otol 2008;122(11):1139-1150

24 Dorafshar AH, Borsuk DE, Bojovic B, et al. Surface anatomy of the middle division of the facial nerve: Zuker's point. Plast Reconstr Surg 2013;131(2):253-257

25 Terzis JK, Tzafetta K. The "babysitter" procedure: minihypoglossal to facial nerve transfer and cross-facial nerve grafting. Plast Reconstr Surg 2009;123(3):865-876

26 Coombs CJ, Ek EW, Wu T, Cleland H, Leung MK. Masseteric-facial nerve coaptation-an alternative technique for facial nerve reinnervation. J Plast Reconstr Aesthet Surg 2009;62(12):1580-1588
27 Manktelow RT, Tomat LR, Zuker RM, Chang M. Smile reconstruction in adults with free muscle transfer innervated by the masseter motor nerve: effectiveness and cerebral adaptation. Plast Reconstr Surg 2006;118(4):885-899

28 Marre D, Hontanilla B. Brain plasticity in Möbius syndrome after unilateral muscle transfer: case report and review of the literature. Ann Plast Surg 2012;68(1):97-100

29 Manni JJ, Beurskens CH, van de Velde C, Stokroos RJ. Reanimation of the paralyzed face by indirect hypoglossal-facial nerve anastomosis. Am J Surg 2001;182(3):268-273

30 Harii K, Ohmori K, Torii S. Free gracilis muscle transplantation, with microneurovascular anastomoses for the treatment of facial paralysis. A preliminary report. Plast Reconstr Surg 1976;57(2): 133-143

31 Takushima A, Harii K, Asato H, Kurita M, Shiraishi T. Fifteen-year survey of one-stage latissimus dorsi muscle transfer for treatment of longstanding facial paralysis. J Plast Reconstr Aesthet Surg 2013; 66(1):29-36

32 Mackinnon SE, Dellon AL. Technical considerations of the latissimus dorsi muscle flap: a segmentally innervated muscle transfer for facial reanimation. Microsurgery 1988;9(1):36-45

33 Harrison DH, Grobbelaar AO. Pectoralis minor muscle transfer for unilateral facial palsy reanimation: an experience of 35 years and 637 cases. J Plast Reconstr Aesthet Surg 2012;65(7): 845-850

34 Terzis JK. Pectoralis minor: a unique muscle for correction of facial palsy. Plast Reconstr Surg 1989;83(5):767-776

35 Dellon AL, Mackinnon SE. Segmentally innervated latissimus dorsi muscle. Microsurgical transfer for facial reanimation. J Reconstr Microsurg 1985;2(1):7-12

36 Yücel A, Aydin Y, Yazar S, Altintaş F, Senyuva C. Elective free-tissue transfer in pediatric patients. J Reconstr Microsurg 2001;17(1):27-36

37 Terzis JK, Olivares FS. Long-term outcomes of free muscle transfer for smile restoration in children. Plast Reconstr Surg 2009;123(2): 543-555

38 Boahene KD, Farrag TY, Ishii L, Byrne PJ. Minimally invasive temporalis tendon transposition. Arch Facial Plast Surg 2011; 13(1):8-13

39 Hussain G, Manktelow RT, Tomat LR. Depressor labii inferioris resection: an effective treatment for marginal mandibular nerve paralysis. Br J Plast Surg 2004;57(6):502-510

40 Tulley P, Webb A, Chana JS, et al. Paralysis of the marginal mandibular branch of the facial nerve: treatment options. $\mathrm{Br} \mathrm{J}$ Plast Surg 2000;53(5):378-385

41 Terzis JK, Kyere SA. Experience with the gold weight and palpebral spring in the management of paralytic lagophthalmos. Plast Reconstr Surg 2008;121(3):806-815

42 Terzis JK, Karypidis D. The outcomes of dynamic procedures for blink restoration in pediatric facial paralysis. Plast Reconstr Surg 2010;125(2):629-644

43 Frey M, Giovanoli P, Tzou CH, Kropf N, Friedl S. Dynamic reconstruction of eye closure by muscle transposition or functional muscle transplantation in facial palsy. Plast Reconstr Surg 2004; 114(4):865-875

44 Manktelow RT, Zuker RM. Muscle transplantation by fascicular territory. Plast Reconstr Surg 1984;73(5):751-757 